Copyright by the AIP Publishing. Heflin, JR; Marciu, D; Figura, C; et al., "Enhanced nonlinear optical response of an endohedral metallofullerene through metal-to-cage charge transfer," Appl. Phys. Lett. 72, 2788 (1998); http:// dx.doi.org/10.1063/1.121456

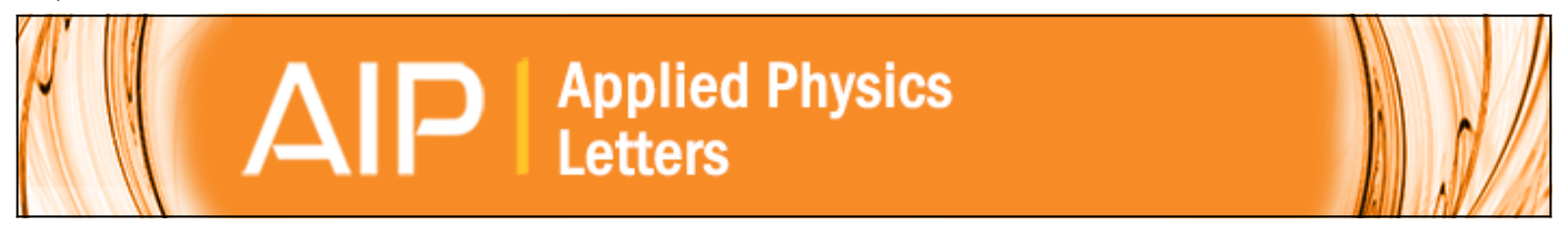

\title{
Enhanced nonlinear optical response of an endohedral metallofullerene through metal- to-cage charge transfer
}

J. R. Heflin, D. Marciu, C. Figura, S. Wang, P. Burbank, S. Stevenson, and H. C. Dorn

Citation: Applied Physics Letters 72, 2788 (1998); doi: 10.1063/1.121456

View online: http://dx.doi.org/10.1063/1.121456

View Table of Contents: http://scitation.aip.org/content/aip/journal/apl/72/22?ver=pdfcov

Published by the AIP Publishing

\section{Articles you may be interested in}

Enhanced nonlinear optical properties of oxygen deficient lead-niobium-germanate film glasses

Appl. Phys. Lett. 90, 251907 (2007); 10.1063/1.2749837

Influence of different peripheral substituents on the nonlinear optical properties of cobalt phthalocyanine core J. Appl. Phys. 101, 083112 (2007); 10.1063/1.2719281

Enhancement of third-order optical nonlinearities by conjugated polymer-bonded carbon nanotubes J. Appl. Phys. 98, 034301 (2005); 10.1063/1.1954887

Nonlinear processes responsible for nondegenerate four-wave mixing in quantum-dot optical amplifiers Appl. Phys. Lett. 77, 1753 (2000); 10.1063/1.1311319

Sub-picosecond resonant third-order nonlinear optical response of azobenzene-doped polymer film J. Appl. Phys. 81, 7073 (1997); 10.1063/1.365270

\section{AlP Re-register for Table of Content Alerts}

\section{Create a profile.

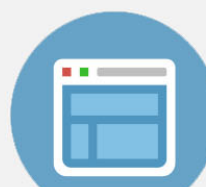

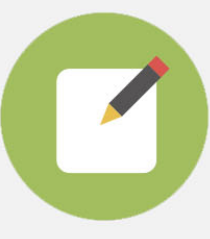




\title{
Enhanced nonlinear optical response of an endohedral metallofullerene through metal-to-cage charge transfer
}

\author{
J. R. Heflin, D. Marciu, C. Figura, and S. Wang \\ Department of Physics, Virginia Tech, Blacksburg, Virginia 24061-0435 \\ P. Burbank, S. Stevenson, and H. C. Dorn \\ Department of Chemistry, Virginia Tech, Blacksburg, Virginia 24061-0212
}

(Received 3 July 1997; accepted for publication 26 March 1998)

\begin{abstract}
A new mechanism for increasing the third-order nonlinear optical susceptibility, $\chi^{(3)}$, is described for endohedral metallofullerenes. A two to three orders of magnitude increase in the nonlinear response is reported for degenerate four-wave mixing experiments conducted with solutions of $\mathrm{Er}_{2} @ C_{82}$ (isomer III) relative to empty-cage fullerenes. A value of $-8.7 \times 10^{-32}$ esu is found for the molecular susceptibility, $\gamma_{x y y x}$, of $\mathrm{Er}_{2} @ C_{82}$ compared to previously reported values of $\gamma_{x x x x}$ $=3 \times 10^{-34}$ esu and $\gamma_{x y y x}=4 \times 10^{-35}$ esu for $C_{60}$. The results confirm the importance of the metal-to-cage charge-transfer mechanism for enhancing the nonlinear optical response in endohedral metallofullerenes. () 1998 American Institute of Physics. [S0003-6951(98)02122-6]
\end{abstract}

Since the initial discovery ${ }^{1}$ and development of a technique for macroscopic preparation ${ }^{2}$ of the archetypal and most abundant fullerene $C_{60}$, a wealth of fullerene-based structures have been produced including higher fullerenes, charge-transfer complexes, fullerene derivatives, superconducting exohedral-doped fullerenes, and carbon nanotubes. One of the most intriguing fullerene classes is the endohedral fullerene in which the spheroidal molecular structure is employed to encapsulate a small number of atoms (one to four) internal to the cage. ${ }^{3-5}$ Until recently, the difficult separation process of endohedral fullerenes had limited their availability to submilligram levels. Consequently, initial studies of these materials had primarily been restricted to electron paramagnetic resonance (EPR) and linear spectroscopy (UV-vis). EPR measurements demonstrated that, for the case of $\mathrm{La} @ C_{82}$, the $\mathrm{La}$ atom transfers three electrons to the fullerene cage and resides in the +3 oxidation state., ${ }^{4,5}$ Metal-to-cage transfer appears to be a common feature of transition metal-containing endohedral metallofullerenes. Meanwhile, nonlinear optical studies of empty-cage $C_{60}$ and $C_{70}$ have shown that these materials possess both large third order susceptibilities $\chi^{(3)}\left(-\omega_{4} ; \omega_{1}, \omega_{2}, \omega_{3}\right)^{6-8}\left(\sim 10^{-11} \mathrm{esu}\right.$ compared to a nonresonant value of $5 \times 10^{-10}$ esu for a polydiacetylene polymer, ${ }^{9}$ for example) and strong optical limiting behavior. ${ }^{10-12}$ We report here the first nonlinear optical measurements of an endohedral metallofullerene and find a dramatic enhancement in the third-order nonlinear optical response. Degenerate four-wave mixing (DFWM) experiments on solutions of the endohedral metallofullerene $\mathrm{Er}_{2} @ C_{82}$ (Fig. 1) show that the metal-to-cage charge transfer provides a mechanism for increasing $\chi^{(3)}\left(-\omega_{4} ; \omega_{1}, \omega_{2}, \omega_{3}\right)$ by orders of magnitude relative to empty-cage fullerenes.

The $\mathrm{Er}_{2} @ C_{82}$ sample was separated and purified using an automated high performance liquid chromatography (HPLC) apparatus that yields multimilligram quantities of pure endohedral metallofullerenes. ${ }^{13-15}$ The initial endohedral fullerene-containing soot was obtained using the Kratschmer-Huffman electric arc burning method on cored graphite rods containing a graphite/metal-oxide mixture. The resulting soot contains both empty-cage fullerenes of varying cage size and primarily the endohedral metallofullerenes $\mathrm{Er} @ C_{82}$ and $\mathrm{Er}_{2} @ C_{82}$ (three isomers). Separation of $\mathrm{Er}_{2} @ C_{82}$ is achieved in a multistep automated HPLC procedure that utilizes Buckyclutcher and Pentabromobenzyl (PBB) derivatized silica gel columns. The inclusion of the PBB chromatographic phase in the separation protocol allows the entire separation process to be automated in contrast with previous procedures. ${ }^{15}$ Three distinct chromatographic fractions are identified by mass spectroscopy as $\mathrm{Er}_{2} @ C_{82}$ indicating multiple isomers of this structure. In the final pass, a single homogeneous chromatographic peak for the latter eluting fraction, $\mathrm{Er}_{2} @ C_{82}$ (isomer III), was obtained utilizing both the PBB and Buckyclutcher columns. The material utilized in this study is purely that identified ${ }^{15}$ as isomer III. The negative-ion mass spectrum for this sample is shown in Fig. 1. An expansion of the mass range centered at $1319 \mathrm{~m} / \mathrm{z}$ is consistent with the predicted isotope distribution for $\mathrm{Er}_{2} @ C_{82}$. The total yield of $\mathrm{Er}_{2} @ C_{82}$ is greater than $1 \%$ of

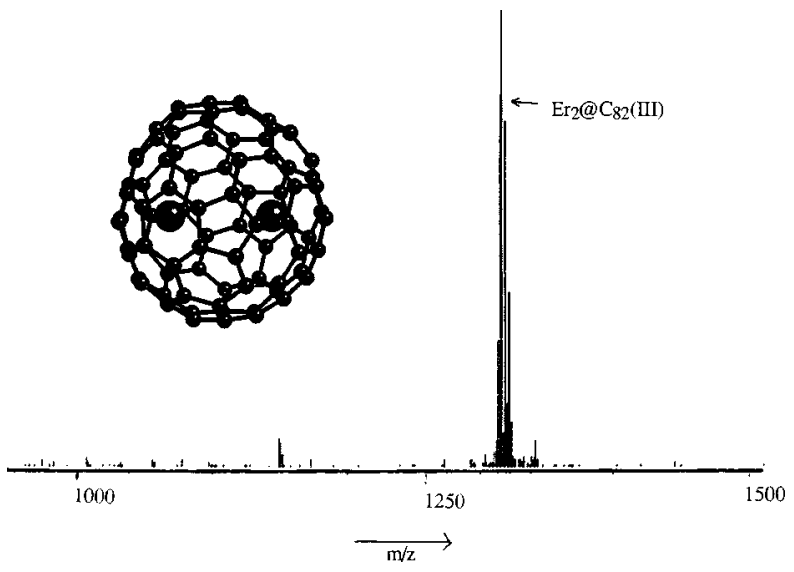

FIG. 1. Negative-ion mass spectrum for purified $\mathrm{Er}_{2} @ C_{82}$ (isomer III) sample. The minor impurity peak at $1152 \mathrm{~m} / \mathrm{z}$ is due to $\operatorname{Er} @ C_{82}$. The computer-generated structure shown in the inset is illustrative and may not reflect the specific cage symmetry and metal sites of the $\mathrm{Er}_{2} @ C_{82}$ isomer. 


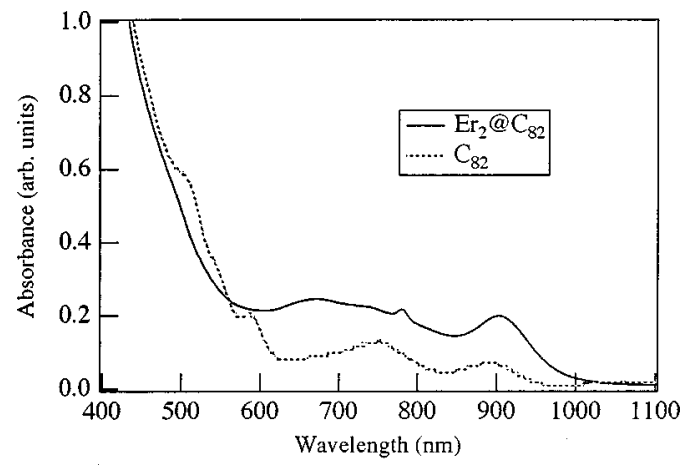

FIG. 2. Absorption spectra of $\mathrm{Er}_{2} @ C_{82}$ and the corresponding empty-cage structure $\mathrm{C}_{82}$ in $\mathrm{CS}_{2}$.

the initial soluble soot which is larger than is typically found for $\mathrm{La}, \mathrm{Sc}$, and $\mathrm{Y}$ extracts.

The absorption spectrum of $\mathrm{Er}_{2} @ C_{82}$ (isomer III) is compared to that of $C_{82}$ in Fig. 2. The endohedral fullerene shows enhanced absorption features near 650 and $900 \mathrm{~nm}$ that result from the charge transfer from the metal to the fullerene cage. The endohedral fullerene also possesses an absorption tail to longer wavelengths in comparison with the empty-cage structure. In both $C_{82}$ and $\mathrm{Er}_{2} @ C_{82}$, there is a very weak residual absorption at $1064 \mathrm{~nm}$, the wavelength at which the DFWM measurements are made. Fluorescence measurements have also been reported in purified $\mathrm{Er}_{2} @ C_{82} \cdot{ }^{16}$ The characteristic $\mathrm{Er}^{3+}$ emission in the $1500 \mathrm{~nm}$ region further demonstrates the occurrence of metal-to-cage charge transfer.

We anticipated the endohedral metallofullerene charge transfer to have a dramatic effect on the nonlinear optical response. It has been shown previously that optical excitation of conjugated molecules can lead to enhancement of $\chi^{(3)}\left(-\omega_{4} ; \omega_{1}, \omega_{2}, \omega_{3}\right)$ by orders of magnitude. This has been demonstrated in both third-harmonic generation measurements ${ }^{17}$ of a naphthalocyanine and in DFWM measurements ${ }^{18,19}$ of a linear polyene. Within the molecular orbital description, the metal-to-cage charge transfer can be considered similar to the effect of optical excitation of the corresponding empty-cage fullerene. In both cases, electrons are placed into the lowest unoccupied molecular orbital (LUMO), though in the metallofullerene there are no corresponding holes produced in the highest occupied molecular orbital (HOMO) as there are in optical excitation. The ground state of the endohedral metallofullerene therefore can be considered to resemble an excited state of the empty cage.

The DFWM experiments are made in the standard, phase-conjugate geometry ${ }^{20}$ using the $1064 \mathrm{~nm}$ fundamental wavelength of a $Q$-switched Nd:YAG laser. The three incident beams are focused to $e^{-2}$ radii of $550 \mu \mathrm{m}$, and the probe beam is at an angle of $5^{\circ}$ with respect to the forward pump beam. A beam splitter is used to direct the signal beam (counterpropagating to the probe beam) to a photodiode to measure its intensity. The maximum energy of the counterpropagating pump beams is $3 \mathrm{~mJ}$ each, corresponding to an intensity of $20 \mathrm{MW} / \mathrm{cm}^{2}$. The sample solutions, with a maximum concentration of $0.32 \mathrm{mg} \mathrm{Er}_{2} @ C_{82}$ in $0.8 \mathrm{ml}$ of $\mathrm{CS}_{2}\left(2.9 \times 10^{-4} \mathrm{~mol} / \mathrm{l}\right)$, are contained in $5 \mathrm{~mm}$ path length spectrophotometer cells. For even the most concentrated solutions, the internal (reflection-corrected) transmittance was

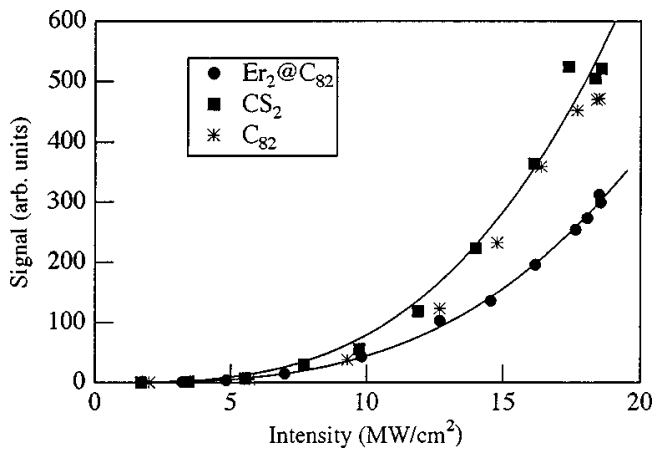

FIG. 3. DFWM signal as a function of incident intensity for $2.4 \times 10^{-4} \mathrm{M}$ $\mathrm{Er}_{2} @ C_{82}$ in $C S_{2}, C_{82}$ in $C S_{2}$, and pure $C S_{2}$ solvent. The solid curves are cubic fits for the $\mathrm{Er}_{2} @ C_{82}$ and $C S_{2}$ data.

greater than $93 \%$ at $1064 \mathrm{~nm}$. In our measurements, the pump beams are vertically polarized while the probe and signal beams are horizontally polarized [thus measuring $\left.\chi_{x y y x}^{(3)}(-\omega ; \omega, \omega,-\omega)\right]$. This configuration ensures that neither thermal nor population gratings contribute to the DFWM signal, since we are interested in the electronic contribution to $\chi_{i j k l}^{(3)}(-\omega ; \omega, \omega,-\omega) .^{21,22}$ This is particularly important since the samples have a small absorption at $1064 \mathrm{~nm}$ corresponding to a molar extinction coefficient $\epsilon$ of $221 \mathrm{M}^{-1} \mathrm{~cm}^{-1}$ and an absorptivity $\alpha$ of $0.15 \mathrm{~cm}^{-1}$ at the highest concentration.

Figure 3 shows the DFWM signal intensity observed for the $C S_{2}$ reference and the $2.4 \times 10^{-4} \mathrm{~mol} / 1 \mathrm{Er}_{2} @ C_{82}$ solution together with fits to the data of the form $A I^{3}$, where $I$ is the incident intensity. A signal dependence on the incident intensity with an alternate exponent would be an indication of undesired mechanisms in the DFWM process, such as two-photon absorption. The DFWM signal [proportional to $\left.\left|\chi_{x y y x}^{(3)}(-\omega ; \omega, \omega,-\omega)\right|^{2}\right]$ of the most concentrated solutions decreases by as much as $50 \%$ relative to pure $C S_{2}$ solvent indicating a very large, negative molecular susceptibility $\gamma_{x y y x}(-\omega ; \omega, \omega,-\omega)$ for $\mathrm{Er}_{2} @ C_{82}$ (isomer III). For a large number of concentrations, the signal is measured as a function of the incident intensity and is fit to a cubic to obtain the $\chi_{x y y x}^{(3)}(-\omega ; \omega, \omega,-\omega)$ value of the solution according to

$$
\begin{aligned}
\chi^{(3)}(-\omega ; \omega, \omega,-\omega)= & \sqrt{\frac{A}{A_{\text {ref }}}}\left(\frac{\alpha l e^{\alpha l / 2}}{1-e^{-\alpha l}}\right) \\
& \times \chi_{\text {ref }}^{(3)}(-\omega ; \omega, \omega,-\omega),
\end{aligned}
$$

where $A$ and $A_{\text {ref }}$ are the coefficients of the cubic fit for the sample and $C S_{2}$ reference, respectively, $l$ is the sample path length, and $\alpha$ is the absorptivity. The value used for the $\mathrm{CS}_{2}$ reference for orthogonally polarized beams is $\chi_{\text {xyyx }}^{(3)}(-\omega ; \omega, \omega,-\omega)=3.6 \times 10^{-13} \mathrm{esu}^{23}$ and the path lengths and refractive indices of the sample and reference are taken to be equal. Figure 4 illustrates the linear decrease of the solution $\chi_{\text {xyyx }}^{(3)}(-\omega ; \omega, \omega,-\omega)$ with increased concentration. From this linear dependence and the relationship

$$
\begin{aligned}
\chi_{\text {solution }}^{(3)}(-\omega ; \omega, \omega,-\omega)= & \chi_{\text {solvent }}^{(3)}(-\omega ; \omega, \omega,-\omega) \\
& +N f^{4} \gamma(-\omega ; \omega, \omega,-\omega),
\end{aligned}
$$

where $N$ is the number density of solute molecules and $f$ is the local field factor $\left(n^{2}+2\right) / 3$ ( $n$ is the refractive index), 


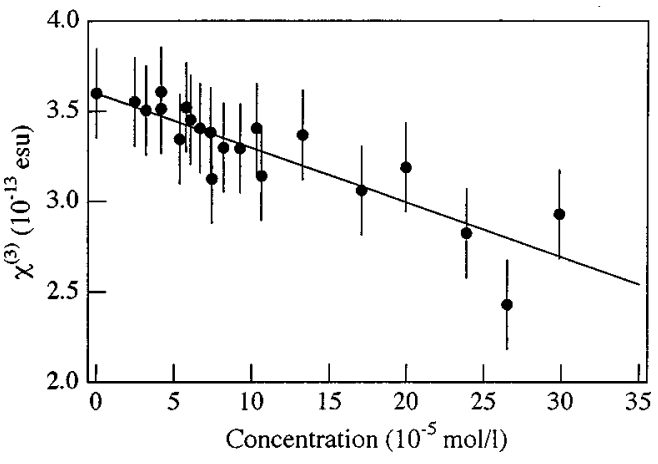

FIG. 4. Concentration dependence of $\chi_{x y y x}^{(3)}(-\omega ; \omega, \omega,-\omega)$ for $\mathrm{Er}_{2} @ C_{82}$ in $C S_{2}$. The uncertainty is derived from the reproducibility in repeated measurements at a fixed concentration.

we obtain a value for the molecular susceptibility, $\gamma_{x y y x}(-\omega ; \omega, \omega,-\omega)$, for $\mathrm{Er}_{2} @ C_{82}$ of $-8.7 \times 10^{-32}$ esu. Using an estimated density of $1.7 \mathrm{gm} / \mathrm{cm}^{3},{ }^{24}$ this corresponds to a $\chi_{x y y x}^{(3)}(-\omega ; \omega, \omega,-\omega)$ for a pure $\mathrm{Er}_{2} @ C_{82}$ film of $-1.1 \times 10^{-9}$ esu. For comparison, in measurements with all beams parallel polarized, values of $\chi_{x x x x}^{(3)}(-\omega ; \omega, \omega,-\omega)$ $=7 \times 10^{-12}$ and $12 \times 10^{-12}$ esu have been previously reported for $C_{60}$ and $C_{70}$ films, respectively, at $1064 \mathrm{~nm}^{6}$ These correspond to respective values for the molecular susceptibility $\gamma_{x x x x}(-\omega ; \omega, \omega,-\omega)$ of $3 \times 10^{-34}$ and 5 $\times 10^{-34}$ esu. For orthogonal polarizations, as in the present work, a value of $\chi_{x y y x}^{(3)}(-\omega ; \omega, \omega,-\omega)=1 \times 10^{-12}$ esu was measured for both films which corresponds to a value for $\gamma_{x y y x}(-\omega ; \omega, \omega,-\omega)$ of $4 \times 10^{-35}$ esu for $C_{60}$.

DFWM was also measured in $C S_{2}$ solutions of the empty-cage fullerenes $C_{60}, C_{82}$, and $C_{84}$ at concentrations equal to or larger than that of the $\mathrm{Er}_{2} @ C_{82}$ solution. In each case, the DFWM signal was indistinguishable from that of the pure solvent demonstrating the substantially smaller values of $\gamma_{x y y x}(-\omega ; \omega, \omega,-\omega)$ in those materials. As an example, the data for $C_{82}$ at a similar concentration to that of $\mathrm{Er}_{2} @ C_{82}$ is shown on Fig. 2. As a further experimental check, the transmittance of the $\mathrm{Er}_{2} @ C_{82}$ solution was measured up to the intensities of the DFWM experiment. The solution behaved as a strictly linear absorber proving that reverse saturable absorption and two-photon absorption have no role in the decrease of the DFWM signal. Finally, we note that a negative-ion mass spectrum was also obtained for the $\mathrm{Er}_{2} @ C_{82}$ sample after all DFWM measurements and is similar to Fig. 1, demonstrating the lack of sample degradation during the nonlinear optical measurements.

The dramatic enhancement in $\chi^{(3)}$ that we have observed in $\mathrm{Er}_{2} @ C_{82}$ establishes the endohedral metallofullerenes as a new potential class of materials for nonlinear optical devices. Specifically, a factor of two to three orders of magnitude higher value for $\chi^{(3)}$ is predicted for a pure film of $\mathrm{Er}_{2} @ C_{82}$ relative to thin films of $C_{60}$ and $C_{70}$, illustrating the importance of metal-to-cage charge transfer as a novel enhancement mechanism. A standard figure of merit for the applicability of materials for nonlinear optical switching and modulation devices is $\chi^{(3)} / \alpha$, where $\alpha$ is the linear absorptivity. As described above, the enhanced $\chi^{(3)}$ observed in $\mathrm{Er}_{2} @ C_{82}$ at $1064 \mathrm{~nm}$ is accompanied by some residual absorption. The absorption is substantially reduced in the 1300 to $1500 \mathrm{~nm}$ optical telecommunications window. Experi- ments are currently underway to measure the nonlinear optical response at these longer wavelengths in both solutions and pure $\mathrm{Er}_{2} @ C_{82}$ films as well as in other endohedral metallofullerenes.

This research was supported in part by a Cottrell Scholars Award of Research Corporation (J.R.H.) and National Science Foundation (H.C.D.). The authors also acknowledge the contribution of erbium endohedral-containing soot provided by D. S. Bethune (IBM, Almaden) and the assistance of Mr. Kim Harick (Virginia Tech) in obtaining the negativeion mass spectrum.

${ }^{1}$ H. W. Kroto, J. R. Heath, S. C. O’Brien, R. F. Curl, and R. E. Smalley, Nature (London) 318, 162 (1985).

${ }^{2}$ W. Kratschmer, L. D. Lamb, K. Fostiropoulos, and D. R. Huffman, Nature (London) 347, 354 (1990).

${ }^{3}$ Y. Chai, T. Guo, C. Jin, R. E. Haufler, L. P. F. Chibante, J. Fure, L. Wang, M. Alford, and R. E. Smalley, J. Phys. Chem. 95, 7564 (1991).

${ }^{4}$ R. D. Johnson, M. S. de Vries, J. Salem, D. S. Bethune, and C. S. Yannoni, Nature (London) 355, 239 (1992).

${ }^{5}$ D. S. Bethune, R. D. Johnson, J. R. Salem, M. S. de Vries, and C. S. Yannoni, Nature (London) 366, 123 (1993).

${ }^{6}$ Z. H. Kafafi, J. R. Lindle, R. G. S. Pong, F. J. Bartoli, L. J. Lingg, and J. Milliken, Chem. Phys. Lett. 188, 492 (1992).

${ }^{7}$ J. S. Meth, H. Vanherzeele, and Y. Wang, Chem. Phys. Lett. 197, 26 (1992).

${ }^{8}$ D. Neher, G. I. Stegeman, F. A. Tinker, and N. Peyghambarian, Opt. Lett. 17, 1491 (1992).

${ }^{9}$ G. M. Carter, M. K. Thakur, Y. J. Chen, and J. V. Hryniewicz, Appl. Phys. Lett. 47, 457 (1985).

${ }^{10}$ L. W. Tutt and A. Kost, Nature (London) 356, 225 (1992).

${ }^{11}$ D. G. McLean, R. L. Sutherland, M. C. Brant, D. M. Brandelik, P. A. Fleitz, and T. Pottenger, Opt. Lett. 18, 858 (1993).

${ }^{12}$ J. R. Heflin, S. Wang, D. Marciu, C. Figura, and R. Yordanov, Proc. SPIE 2530, 176 (1995).

${ }^{13}$ S. Stevenson, H. C. Dorn, P. Burbank, K. Harich, Z. Sun, C. H. Kiang, J. R. Salem, M. S. de Vries, P. H. M. van Loosdrecht, R. D. Johnson, and C. S. Yannoni, Anal. Chem. 66, 2680 (1994).

${ }^{14}$ S. Stevenson, H. C. Dorn, P. Burbank, K. Harich, J. Haynes, C. H. Kiang, J. R. Salem, M. S. de Vries, P. H. M. van Loosdrecht, R. D. Johnson, C. S. Yannoni, and D. S. Bethune, Anal. Chem. 66, 2675 (1994).

${ }^{15}$ H. C. Dorn, S. Stevenson, P. Burbank, Z. Sun, T. Glass, K. Harich, P. H. M. van Loosdrecht, R. D. Johnson, R. Beyers, J. R. Salem, M. S. de Vries, C. S. Yannoni, C. H. Kiang, and D. S. Bethune, Mater. Res. Soc. Symp. Proc. 359, 123 (1995).

${ }^{16}$ R. M. MacFarlane, G. Wittman, P. H. M. van Loosdrecht, M. de Vries, D. S. Bethune, S. Stevenson, and H. C. Dorn, Phys. Rev. Lett. 79, 1397 (1997).

${ }^{17}$ J. R. Heflin, D. C. Rodenberger, R. F. Shi, M. Wu, N. Q. Wang, Y. M. Cai, and A. F. Garito, Phys. Rev. A 45, R4233 (1992).

${ }^{18}$ D. C. Rodenberger, J. R. Heflin, and A. F. Garito, Nature (London) 359, 309 (1992).

${ }^{19}$ D. C. Rodenberger, J. R. Heflin, and A. F. Garito, Phys. Rev. A 51, 3234 (1995).

${ }^{20}$ See, for example, B. E. A. Saleh and M. C. Teich, Fundamentals of Photonics (Wiley, New York, 1991).

${ }^{21}$ J. Etchepare, G. Grillon, J. P. Chambaret, G. Hamoniaux, and A. Orszag, Opt. Commun. 63, 329 (1987).

${ }^{22}$ P. A. Fleitz, R. L. Sutherland, L. V. Natarajan, T. Pottenger, and N. C. Fernelius, Opt. Lett. 17, 716 (1992).

${ }^{23}$ R. W. Hellwarth, Prog. Quantum Electron. 5, 1 (1977).

${ }^{24}$ P. W. Stephens, L. Mihaly, P. L. Lee, R. L. Whetten, S. M. Huang, R. Kaner, F. Deiderich, and K. Holczer, Nature (London) 351, 632 (1991). 